\title{
Combined Minivalley and Layer Control in Twisted Double Bilayer Graphene
}

\section{Journal Article}

\section{Author(s):}

de Vries, Folkert Kornelis; Zhu, Jihang; Portoles, Elias; Zheng, Giulia; Masseroni, Michele; Kurzmann, Annika; Taniguchi, Takashi; Watanabe, Kenji; MacDonald, Allan H.; Ensslin, Klaus (D); Ihn, Thomas Markus (D); Rickhaus, Peter (D)

Publication date:

2020-10-23

Permanent link:

https://doi.org/10.3929/ethz-b-000449000

Rights / license:

In Copyright - Non-Commercial Use Permitted

Originally published in:

Physical Review Letters 125(17), https://doi.org/10.1103/PhysRevLett.125.176801 


\title{
Combined Minivalley and Layer Control in Twisted Double Bilayer Graphene
}

\author{
Folkert K. de Vries $\odot,{ }^{1,}{ }^{*}$ Jihang Zhu, ${ }^{2}$ Elías Portolés $\odot,{ }^{1}$ Giulia Zheng $\odot,{ }^{1}$ Michele Masseroni, ${ }^{1}$ Annika Kurzmann, ${ }^{1}$ \\ Takashi Taniguchi, ${ }^{3}$ Kenji Watanabe $\odot,{ }^{3}$ Allan H. MacDonald, ${ }^{2}$ Klaus Ensslin $\odot,{ }^{1}$ Thomas Ihn, ${ }^{1}$ and Peter Rickhaus $\oplus^{1, \dagger}$ \\ ${ }^{1}$ Laboratory for Solid State Physics, ETH Zürich, CH-8093 Zürich, Switzerland \\ ${ }^{2}$ Department of Physics, The University of Texas at Austin, Austin, Texas 78712, USA \\ ${ }^{3}$ National Institute for Material Science, 1-1 Namiki, Tsukuba 305-0044, Japan
}

(Received 12 February 2020; accepted 3 September 2020; published 20 October 2020)

\begin{abstract}
Control over minivalley polarization and interlayer coupling is demonstrated in double bilayer graphene twisted with an angle of $2.37^{\circ}$. This intermediate angle is small enough for the minibands to form and large enough such that the charge carrier gases in the layers can be tuned independently. Using a dual-gated geometry we identify and control all possible combinations of minivalley polarization via the population of the two bilayers. An applied displacement field opens a band gap in either of the two bilayers, allowing us to even obtain full minivalley polarization. In addition, the carriers, formerly separated by their minivalley character, are mixed by tuning through a Lifshitz transition, where the Fermi surface topology changes. The high degree of control over the minivalley character of the bulk charge transport in twisted double bilayer graphene offers new opportunities for realizing valleytronics devices such as valley valves, filters, and logic gates.
\end{abstract}

DOI: 10.1103/PhysRevLett.125.176801

The band structure of graphene exhibits two minima at the same energy known as "valleys." These valleys could form the basis for a fundamentally new type of electronics $[1,2]$. Experimental realizations of such valleytronic devices include valley polarized transport [3], the valley Hall effect [4] or a valley valve [5]. Recently, twisting two graphene sheets has opened up a new family of twodimensional hexagonal systems having a valley degree of freedom as well. The twist leads to a superlattice, or moiré pattern, which introduces a new unit cell in real space [Fig. 1(a)] and a new or mini unit cell in reciprocal space with its own minivalleys [Fig. 1(b)] that has remarkable effects on the band structure [6]. At small twist angles, below $1^{\circ}$, the layers are coupled and the superlattice leads to insulating regions with different topology [7], resulting in topological edge channels between these regions [8]. For angles of $\sim 1^{\circ}$, isolated flat bands arise in the coupled layers for twisted bilayer graphene (TBG) [9] and the bands are flattened in twisted double bilayer graphene (TDBG) $[10,11]$. Flattening of bands boosts many-body interactions and leads to the formation of correlated states such as insulators and superconductors. These states have been measured in TBG $[12,13]$. Recently, correlated states were observed in TDBG as well [14-16]. For large twist angles $\left(\sim 10^{\circ}\right.$ or larger) the layers are decoupled [17]. The charge carrier wave functions are then bound to one of the two layers only, and can therefore be tuned independently using a dual-gated structure [17,18]. While the large and small angle devices have been studied thoroughly, the angles in between $\left(\sim 2^{\circ}\right)$ have received limited attention in experiments [19-21]. Potential correlated states aside, these intermediate angles are relevant in terms of layer and minivalley tunability since the energy of the crossover regime between coupled and decoupled wave functions can be accessed by gating.

Our TDBG device with an angle of $2.37^{\circ}$ [Fig. 1(c)] exhibits a band structure resembling bilayer graphene but on a much smaller energy scale, as shown in Fig. 1(d). A global bottom gate and top gate enable us to tune the density and displacement field in the two bilayers, creating a tunable test bed to explore the minibands and minivalleys. The displacement field controls the band gaps in each of the bilayers $[17,23]$. The different electronic states expected from band structure calculations are introduced in Figs. 1(d) and 1(e), sorted by the characteristic point in the miniBrillouin zone $\left(\kappa, \kappa^{\prime}\right.$, or $\left.\gamma\right)$ enclosed by the Fermi surfaces. The $\kappa$ - and $\kappa^{\prime}$ states are mostly confined to one bilayer each and therefore decoupled. This decoupling provides control over the layer population via the electrostatic top and bottom gates. Since the carriers in the decoupled bilayers are minivalley polarized, the gates directly control the minivalley character of the bulk transport in the device. Such gate control over the (mini)valley degree of freedom separates TDBG from graphene devices. Perfect minivalley polarization can be achieved when the Fermi level is tuned into the band gap at $\kappa$ or $\kappa^{\prime}$ by tuning the displacement field. This is in contrast with TBG where only partial minivalley polarization can be reached [24]. Contrary to $\kappa$ and $\kappa^{\prime}$ states, carriers stemming from the $\gamma$ points occupy all layers [Fig. 1(e)], i.e., the two bilayers are coupled. Tuning the Fermi level to these $\gamma$ states involves going through a Lifshitz transition, a change in the Fermi surface topology [25], where the wave functions cross 
(a)

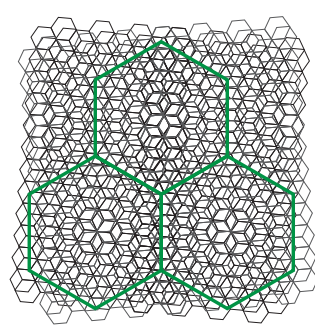

(c)

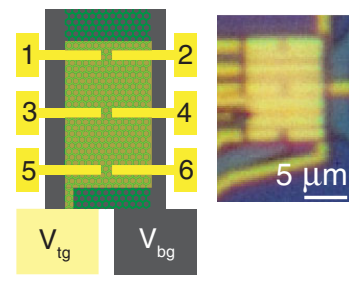

(b)

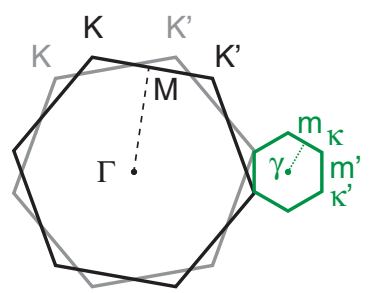

(d)

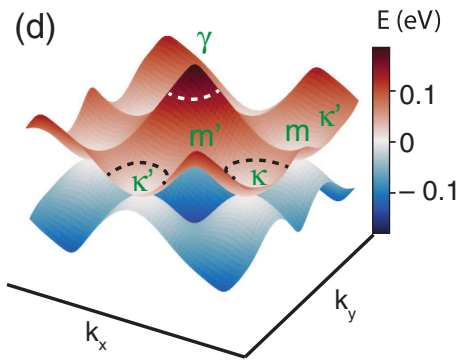

(e)

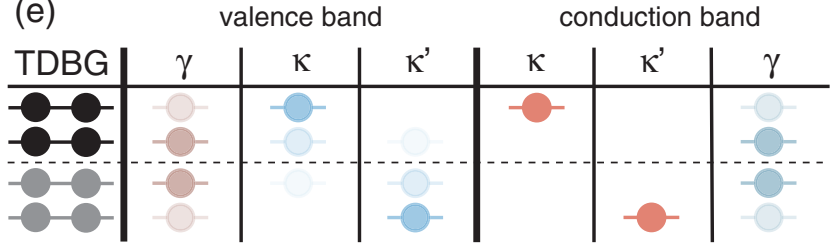

FIG. 1. (a) Schematic representation of twisted double bilayer graphene (TDBG), forming moiré unit cells indicated by the green hexagons. (b) Sketch of the Brillouin zone (BZ) of the two bilayers in TDBG (gray and black), leading to the definition of a mini-BZ (green). The characteristic points of the mini-BZs are indicated. (c) The left panel depicts the device measured, with TDBG (green), graphite bottom gate $V_{\mathrm{bg}}$ (dark gray), global top gate $V_{\text {tg }}$ (opaque yellow), and numbered Ohmic contacts (yellow). The right panel shows an optical image of the device. (d) Lowest energy (first moiré) valence and conduction band of TDBG with a twist of $2.3^{\circ}$, calculated using a continuum model described in the Supplemental Material [22]. The characteristic points of the mini-BZ are indicated and Fermi contours around these points are sketched with a dotted line. (e) Schematic representation of the calculated wave function probability distribution over the four graphene layers for the conduction and valence band at zero displacement field, labeled by the origin of their Fermi surfaces in reciprocal space. The color represents the charge (electrons are red, holes are blue) and the opacity the probability. For details see the Supplemental Material [22].

over from populating a single bilayer, to being spread over all layers [Fig. 1(e)]. This allows us to mix the carriers that were formerly separated by their minivalley character. To summarize, by gating, carriers residing in the minivalleys can be addressed individually, filtered, and mixed. Such minivalley control makes TDBG with an intermediate angle a promising platform for a new efficient type of electronics, called valleytronics.

Here, we use high quality TDBG devices to investigate the tunable minivalley polarization and interlayer coupling at a twist angle of $2.37^{\circ}$. Using a dual-gated structure, we first map out the different minivalley states by measuring
Shubnikov-de Haas $(\mathrm{SdH})$ oscillations as a function of total density and displacement field. We analyze the measurements using a capacitance model and band structure calculations. Then, we interpret the transition between coupled and decoupled layers in terms of a Lifshitz transition.

We twist two graphene bilayers using the tear and stack method [26], and fabricate a multiterminal device as shown in Fig. 1(c) (for details see Supplemental Material [22]). The TDBG stack is sandwiched between two layers of hexagonal boron nitride ( $\mathrm{hBN})$. On one side a graphite layer, used as a global bottom gate $V_{\mathrm{bg}}$, is added. The entire stack is transferred onto a $\mathrm{Si} / \mathrm{SiO}_{2}(285 \mathrm{~nm})$ substrate. We define the TDBG mesa by reactive ion etching, and evaporate $\mathrm{Cr} / \mathrm{Au}$ to form the Ohmic edge contacts [yellow in Fig. 1(c)]. Then two layers of top gates, separated by an aluminum oxide dielectric, are deposited on top of the stack. Throughout this manuscript we bias the two top gates such that they together act as a single global top gate $V_{\mathrm{tg}}$. All measurements are performed in a dilution cryostat at a temperature of $70 \mathrm{mK}$, unless stated otherwise. We use a two-terminal voltage bias setup to obtain the conductance $G$, or a four-terminal current bias setup to measure the longitudinal and Hall resistances, $R_{x x}$ and $R_{x y}$, both with standard lock-in techniques (see Supplemental Material [22]). The magnetic field is applied perpendicular to the plane of the sample. The sample contains three similar devices, one of which is used in the main text, the other two, as well as other contact configuration of the first, are presented in the Supplemental Material [22].

To study the phase diagram of our TDBG device we present the conductance modulation $\Delta G$, after subtracting a smooth background from the measured conductance $G$, as a function of $V_{\mathrm{tg}}$ and $V_{\mathrm{bg}}$ at a magnetic field $B=2 \mathrm{~T}$ in Fig. 2(a). Graphs of the conductance at various $B$ and details on the background subtraction are presented in the Supplemental Material [22], as well as an estimation of the carrier mobility of $\sim 25.000 \mathrm{~cm}^{2} / \mathrm{V} \mathrm{s}$ and a mean free path of $\sim 350 \mathrm{~nm}$. For clarity we depicted the origin and directions [yellow arrows in Fig. 2(b)] of the total density $n \propto C_{\mathrm{tg}} V_{\mathrm{tg}}+C_{\mathrm{bg}} V_{\mathrm{bg}}$ and displacement field $D \propto C_{\mathrm{bg}} V_{\mathrm{bg}}-$ $C_{\mathrm{tg}} V_{\mathrm{tg}}$, respectively, where $C_{i}$ is the capacitance between the TDBG and gate electrode $i$. The $\mathrm{SdH}$ oscillations in Fig. 2(a) reveal two types of regions. First, regions with a single set of $\mathrm{SdH}$ oscillations along constant $n$ show up (e.g., around $V_{\mathrm{bg}}, V_{\mathrm{tg}}=8 \mathrm{~V},-10 \mathrm{~V}$ ), implying the existence of a single carrier gas of density $n$. Second, we observe regions with two sets of $\mathrm{SdH}$ oscillations that cross each other (e.g., around $V_{\mathrm{bg}}, V_{\mathrm{tg}}=4 \mathrm{~V}, 4 \mathrm{~V}$ ). We interpret this as having two decoupled carrier gases occupying the two separate bilayers, one that is coupled more strongly to the top gate and one that is coupled more strongly to the bottom gate. The overall asymmetry with respect to the $D$ axis is caused by a combination of the inherent electronhole asymmetry of TDBG [10] and the recently described 


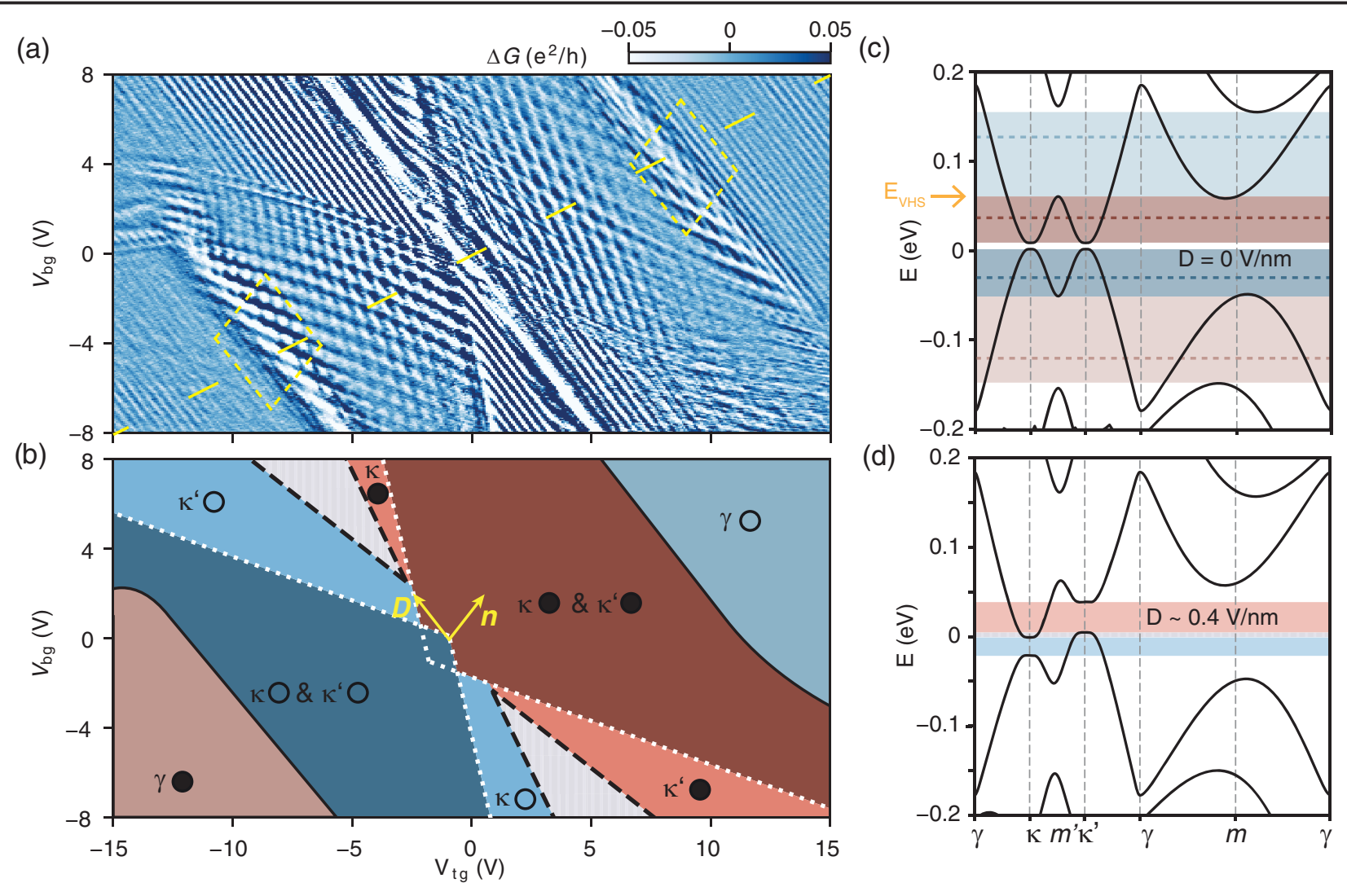

FIG. 2. (a) The conductance modulation $\Delta G$, measured between contacts 1 and 2 and between contacts 3 and 4 [Fig. $1(\mathrm{~d})$ ] shows Shubnikov-de Haas oscillations as a function of top gate $V_{\mathrm{tg}}$ and bottom gate $V_{\mathrm{bg}}$, at a magnetic field $B=2 \mathrm{~T}$. Gate voltages used in Figs. 3(a)-3(c) are plotted with the dashed yellow line along the diagonal, and the dashed yellow boxes, respectively. (b) Phase diagram of the carriers present as a function of $V_{\mathrm{tg}}$ and $V_{\mathrm{bg}}$. The dotted white and dashed black lines are calculated using the capacitor model discussed in Ref. [27] and the solid black lines are sketched. The regions are colored according to the electronic states being occupied (red) or empty (blue), and the characteristic point from which the Fermi surfaces originate. The directions of the total density $n$ and displacement field $D$ are displayed by the yellow arrows. (c),(d) Band structure calculations at zero $D$ (c) and $D \sim 0.4 \mathrm{~V} / \mathrm{nm}$ (d). The color of the highlighted energy windows correspond to the regions indicated in (b). The energy at which a van Hove singularity occurs $E_{\mathrm{VHS}}$ at the $m^{\prime}$ point is highlighted by an orange arrow. See the Supplemental Material for details on the calculations [22].

built-in crystal field [17]. We sketch the different regions of the phase diagram in Fig. 2(b). In order to identify on which layers and minivalleys the carriers reside in the different regions we compare the data to a capacitor model and band structure calculations.

The results of the capacitor model are presented as the dotted white and dashed black lines in Fig. 2(b) (for details see Supplemental Material [22]), which represent the coincidence of the Fermi energy $E_{\mathrm{F}}$ with band extrema of parabolic electron and hole minibands at $\kappa$ and $\kappa^{\prime}$. These lines separate different regions of band population, colored according to the electronic states being occupied (electrons) or empty (holes), red and blue, respectively. In the dark red and blue regions both $\kappa$ and $\kappa^{\prime}$ minibands are populated, resembling the regions having two sets of $\mathrm{SdH}$ oscillations with different slopes in Fig. 2(a). The coupling [slopes in Figs. 2(a) and 2(b)] of the two carrier gases to $V_{\mathrm{tg}}$ and $V_{\mathrm{bg}}$ differs because their wave functions are mostly localized in separate layers [Fig. 1(e)], causing one of the two gates to be screened by the carriers in the other layer. The light red and blue regions [see also Fig. 2(d)] highlight the parts where only one of the two minibands $\left(\kappa\right.$ or $\left.\kappa^{\prime}\right)$ is populated due to the finite $D$ that both increases the band gaps $\Delta_{D}$ and shifts them with respect to each other. Since only the band at $\kappa$ or $\kappa^{\prime}$ is populated, the device is completely minivalley polarized in these regimes. This is in accordance to the single set of $\mathrm{SdH}$ oscillations parallel to $D$ observed in Fig. 2(a). There, the Fermi energy $E_{F}$ in the other layer resides in the gap, such that no screening occurs. The gray area enclosed by the dashed black lines represents overlap of electron and hole minibands from $\kappa$ and $\kappa^{\prime}$, and vice versa. In this region the single particle picture breaks down. Therefore, it is outside the scope of this manuscript, but is treated in detail in Ref. [27]. Up to this point, we identified several minivalley polarized (layer decoupled) states, all based on the wave function of the carriers at $\kappa$ and $\kappa^{\prime}$ [Fig. 1(e)]. Electrons or holes are present in either of the two minivalleys separately, both minivalleys can be populated 
with the same carrier type, or they can be populated with different carrier types. We thus have full control over the minivalley polarization of the bulk charge transport in our TDBG device.

When tuning from charge neutrality to larger $E_{F}$ (and $n$ ) we expect to enter a regime where the carriers at $E_{F}$ encircle the $\gamma$ point [Fig. 1(d)]. The wave function of the $\gamma$ carriers is spread over all four layers as sketched in Fig. 1(e) and therefore the two bilayers are coupled. Indeed, we do observe a single set of SdH oscillations in Fig. 2(a) above a certain density threshold. Therefore, we hypothesize that a Lifshitz transition, a change of the Fermi surface topology, occurs along the solid black line in Fig. 2(b). Such a Lifshitz transition has been observed before in TBG $[20,21]$. We extend the validity and range of these pioneering works by adding independent control over displacement field and density.

For further understanding, the band structure is calculated using a continuum model, where linear potentials between the layers are used to model the displacement field. A crystal field of $0.1 \mathrm{~V} / \mathrm{nm}$ is included [17], leading to a total gap at zero $D$ of $\sim 7 \mathrm{meV}$. The different carrier regions discussed before are apparent in the band structures calculated at $D=0 \mathrm{~V} / \mathrm{nm}$ and $D \sim 0.4 \mathrm{~V} / \mathrm{nm}$ in Figs. 2(c) and 2(d). We observe that the parabolic band picture is limited to a Fermi energy $E_{F}$ of at most half of the energy of the van Hove singularity $E_{\mathrm{VHS}}$ at the $m^{\prime}$ and $m$ point [19]. Also, at $E_{F}=E_{\mathrm{VHS}}$ the center(s) of the Fermi surface(s) changes from the $\kappa$ and $\kappa^{\prime}$ points to the $\gamma$ point. The Lifshitz transition should thus involve a change in both between electrons and holes, and degeneracy.

In Fig. 3(a) we present the longitudinal resistance $R_{x x}$ times the sign of the Hall resistance $R_{x y}$ as a function of $B$ while changing $n$ and $D$ at the same time, spanning the diagonal of the gate-gate map in Fig. 2(a) (see Supplemental Material for constant $D$ measurements). The color thus represents the carrier type (red for electrons, blue for holes) and the contrast is given by $R_{x x}$. We observe changes in the sign of $R_{x y}$ implying a crossover between electrons and holes, and Landau level fans of the corresponding electron and hole Fermi surfaces. The Landau fans emerging at nonzero densities originate from the fully filled first moiré band. Apart from the expected change in carrier type at the charge neutrality point $(n=0)$ we observe two more sign changes, at $n=-5.25 \times$ $10^{12} \mathrm{~cm}^{-2}$ and $n=5.6 \times 10^{12} \mathrm{~cm}^{-2}$. At these densities the electron and hole Landau fans emerging from different points ( $\kappa$ and $\kappa^{\prime}$, and $\gamma$, respectively) cross. Using the densities where the Landau fans emerge and where they cross (corresponding to $E_{\mathrm{VHS}}$ ) we estimate the twist angle of $2.3^{\circ}$, which we are able to refine with the Hofstadter butterfly spectrum to $2.37^{\circ}$, see Supplemental Material [22]. Additionally, at the point where $R_{x y}$ changes sign, the degeneracy changes by a factor 2 for a trace at constant $D$. The periodicity of the $\mathrm{SdH}$ oscillations as a function of $n$
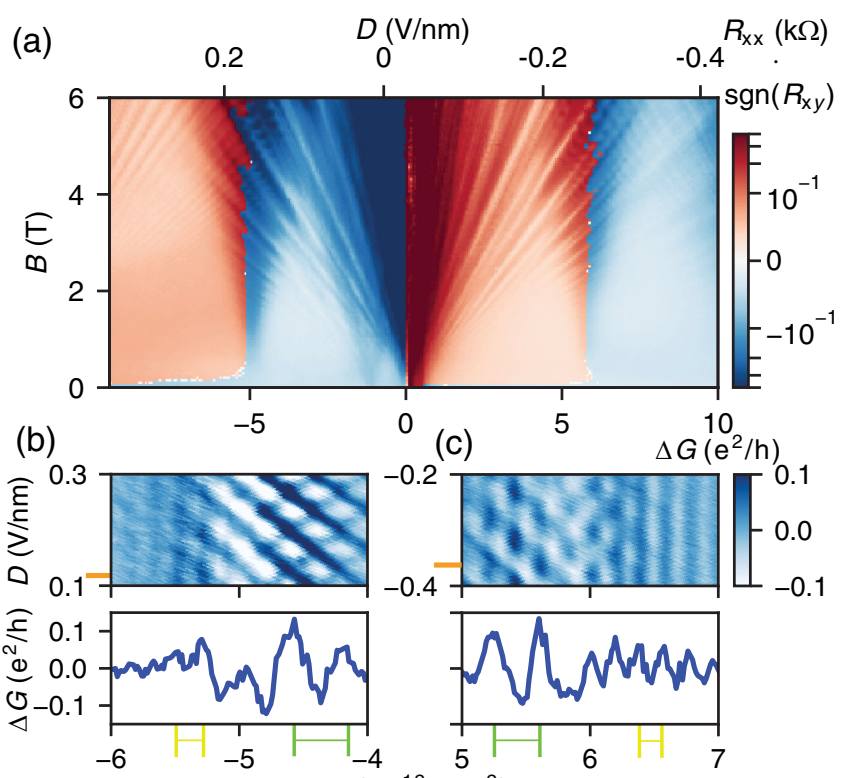

(c)
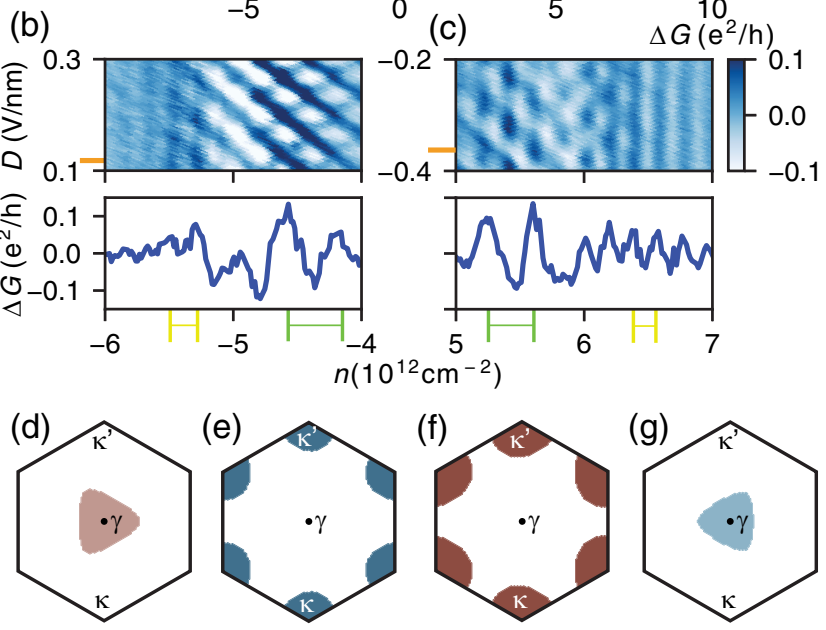

FIG. 3. (a) Longitudinal resistance $R_{x x}$ measured between contacts 2 and 4 times the sign of the Hall resistance $R_{x y}$ measured between contacts 3 and 4 with a current applied from contact 1 to 6 , as a function of density $n$ and displacement field $D$, and magnetic field $B$. A symmetric logarithmic color scale is used, where the section around zero is linearly interpolated. (b),(c) $\Delta G$ as a function of $D$ and $n$, including a line trace obtained by binning the data along the $D$ axis, at a value indicated by the orange ticks. The change in oscillation period is indicated by the green and yellow spacers. (d)-(g) Fermi surfaces drawn in the mini-Brillouin zone calculated at $D=0 \mathrm{~V} / \mathrm{nm}$ and energies as indicated in Fig. 2(c). The closed surfaces are colored following Fig. 2(b).

[indicated by the yellow and green spacers in Figs. 3(b) and 3(c)] provides us with $\delta n=1.9 \times 10^{11} \mathrm{~cm}^{-2}$ and $\delta n=3.9 \times 10^{11} \mathrm{~cm}^{-2}$. This change in periodicity of the Landau levels as a function of density corresponds to a change in degeneracy from $\sim 4$ to $\sim 8$, respectively, where a degeneracy of 4 stems from the spin and valley degrees of freedom in bilayer graphene, and the factor 2 stems from the layer degeneracy. We thus confirm the Lifshitz transition, where the Fermi surface changes from two electronhole surfaces around $\kappa$ and $\kappa^{\prime}$ to a single hole-electron Fermi surface around $\gamma$. We illustrate this by presenting the calculated Fermi surfaces for $D=0$ in Figs. 3(d)-3(g). We can thus either populate states where the two bilayers are decoupled and individually controllable, or where they are coupled, leading to a single four-layer system [Fig. 1(e)]. Therefore, we can not only address the minivalleys 
individually but also mix the corresponding wave functions by passing through the Lifshitz transition. In addition, locally tuning across the Lifshitz transition can potentially be exploited to control the valley degree of freedom [28].

In conclusion, we have used dual-gated TDBG with an intermediate twist angle to demonstrate control over the minivalley polarization and the interlayer coupling. Using local gates to select $\kappa^{-}, \kappa^{\prime}$ character in the bulk charge transport enables future engineering of valley valves and filters [28]. Perfect valley polarization in the bulk offers greater device flexibility in comparison to realizations where the valley polarized transport only occurs at natural edges or topological boundaries. Combining this with the empty states and states with $\gamma$ character, opens the possibility to develop logic gates based on the valley degree freedom [29]. These potential applications show that TDBG with an intermediate angle is a promising route towards valleytronics [1,2].

The data used in this Letter is made available online [30].

We acknowledge the support of the ETH FIRST laboratory, and financial support from the European Graphene Flagship and the Swiss National Science Foundation via NCCR Quantum Science. P.R. acknowledges financial support from the ETH Fellowship program. Growth of hexagonal boron nitride crystals was supported by the Elemental Strategy Initiative conducted by MEXT, Japan and the CREST (JPMJCR15F3), JST.

*devriesf@phys.ethz.ch †peterri@phys.ethz.ch

[1] A. Rycerz, J. Tworzydlo, and C. W. J. Beenakker, Valley filter and valley valve in graphene, Nat. Phys. 3, 172 (2007).

[2] J. R. Schaibley, H. Yu, G. Clark, P. Rivera, J. S. Ross, K. L. Seyler, W. Yao, and X. Xu, Valleytronics in 2D materials, Nat. Rev. Mater. 1, 16055 (2016).

[3] L. Ju, Z. Shi, N. Nair, Y. Lv, C. Jin, J. Velasco, C. OjedaAristizabal, H. A. Bechtel, M. C. Martin, A. Zettl, J. Analytis, and F. Wang, Topological valley transport at bilayer graphene domain walls, Nature (London) 520, 650 (2015).

[4] R. V. Gorbachev, J. C. W. Song, G. L. Yu, A. V. Kretinin, F. Withers, Y. Cao, A. Mishchenko, I. V. Grigorieva, K. S. Novoselov, L. S. Levitov, and A. K. Geim, Detecting topological currents in graphene superlattices, Science 346, 448 (2014).

[5] J. Li, R.-X. Zhang, Z. Yin, J. Zhang, K. Watanabe, T. Taniguchi, C. Liu, and J. Zhu, A valley valve and electron beam splitter, Science 362, 1149 (2018).

[6] R. Bistritzer and A. H. MacDonald, Moiré bands in twisted double-layer graphene, Proc. Natl. Acad. Sci. U.S.A. 108, 12233 (2011).

[7] P. San-Jose and E. Prada, Helical networks in twisted bilayer graphene under interlayer bias, Phys. Rev. B 88, 121408(R) (2013).

[8] P. Rickhaus, J. Wallbank, S. Slizovskiy, R. Pisoni, H. Overweg, Y. Lee, M. Eich, M.-H. Liu, K. Watanabe,
T. Taniguchi, T. Ihn, and K. Ensslin, Transport through a network of topological channels in twisted bilayer graphene, Nano Lett. 18, 6725 (2018).

[9] E. Suárez Morell, J. D. Correa, P. Vargas, M. Pacheco, and Z. Barticevic, Flat bands in slightly twisted bilayer graphene: Tight-binding calculations, Phys. Rev. B 82, 121407(R) (2010).

[10] J. Y. Lee, E. Khalaf, S. Liu, X. Liu, Z. Hao, P. Kim, and A. Vishwanath, Theory of correlated insulating behaviour and spin-triplet superconductivity in twisted double bilayer graphene, Nat. Commun. 10, 5333 (2019).

[11] J. Liu, Z. Ma, J. Gao, and X. Dai, Quantum Valley Hall Effect, Orbital Magnetism, and Anomalous Hall Effect in Twisted Multilayer Graphene Systems, Phys. Rev. X 9, 031021 (2019).

[12] Y. Cao, V. Fatemi, A. Demir, S. Fang, S. L. Tomarken, J. Y. Luo, J. D. Sanchez-Yamagishi, K. Watanabe, T. Taniguchi, E. Kaxiras, R. C. Ashoori, and P. Jarillo-Herrero, Correlated insulator behaviour at half-filling in magic-angle graphene superlattices, Nature (London) 556, 80 (2018).

[13] Y. Cao, V. Fatemi, S. Fang, K. Watanabe, T. Taniguchi, E. Kaxiras, and P. Jarillo-Herrero, Unconventional superconductivity in magic-angle graphene superlattices, Nature (London) 556, 43 (2018b).

[14] G. W. Burg, J. Zhu, T. Taniguchi, K. Watanabe, A. H. MacDonald, and E. Tutuc, Correlated Insulating States in Twisted Double Bilayer Graphene, Phys. Rev. Lett. 123, 197702 (2019).

[15] C. Shen, Y. Chu, Q. Wu, N. Li, S. Wang, Y. Zhao, J. Tang, J. Liu, J. Tian, K. Watanabe, T. Taniguchi, R. Yang, Z. Y. Meng, D. Shi, O. V. Yazyev, and G. Zhang, Correlated states in twisted double bilayer graphene, Nat. Phys. 16, 520 (2020).

[16] X. Liu, Z. Hao, E. Khalaf, J. Y. Lee, Y. Ronen, H. Yoo, D. H Najafabadi, K. Watanabe, T.Taniguchi, A. Vishwanath, and P. Kim, Tunable spin-polarized correlated states in twisted double bilayer graphene, Nature (London) 583, 221 (2020).

[17] P. Rickhaus, G. Zheng, J. L. Lado, Y. Lee, A. Kurzmann, M. Eich, R. Pisoni, C. Tong, R. Garreis, C. Gold, M. Masseroni, T. Taniguchi, K. Wantanabe, T. Ihn, and K. Ensslin, Gap opening in twisted double bilayer graphene by crystal fields, Nano Lett. 19, 8821 (2019).

[18] P. Rickhaus, M.-H. Liu, M. Kurpas, A. Kurzmann, Y. Lee, H. Overweg, M. Eich, R. Pisoni, T. Taniguchi, K. Watanabe, K. Richter, K. Ensslin, and T. Ihn, The electronic thickness of graphene, Sci. Adv. 6, eaay8409 (2020).

[19] G. Li, A. Luican, J. M. B. Lopes dos Santos, A. H. Castro Neto, A. Reina, J. Kong, and E. Y. Andrei, Observation of van Hove singularities in twisted graphene layers, Nat. Phys. 6, 109 (2010).

[20] Y. Kim, P. Herlinger, P. Moon, M. Koshino, T. Taniguchi, K. Watanabe, and J. H. Smet, Charge inversion and topological phase transition at a twist angle induced van Hove singularity of bilayer graphene, Nano Lett. 16, 5053 (2016).

[21] Y. Cao, J. Y. Luo, V. Fatemi, S. Fang, J. D. SanchezYamagishi, K. Watanabe, T. Taniguchi, E. Kaxiras, and P. Jarillo-Herrero, Superlattice-Induced Insulating States and Valley-Protected Orbits in Twisted Bilayer Graphene, Phys. Rev. Lett. 117, 116804 (2016). 
[22] See Supplemental Material at http://link.aps.org/ supplemental/10.1103/PhysRevLett.125.176801 for details on the band structure calculation, angle extraction and additional data of devices 2 and 3 .

[23] J. B. Oostinga, H. B. Heersche, X. Liu, A. F. Morpurgo, and L. M. K. Vandersypen, Gate-induced insulating state in bilayer graphene devices, Nat. Mater. 7, 151 (2008).

[24] A. I. Berdyugin, B. Tsim, P. Kumaravadivel, S. G. Xu, A. Ceferino, A. Knothe, R. K. Kumar, T. Taniguchi, K. Watanabe, A. K. Geim, I. V. Grigorieva, and V. I. Fal'ko, Minibands in twisted bilayer graphene probed by magnetic focusing, Sci. Adv. 6, eaay7838 (2020).

[25] I. M. Lifshitz, Anomalies of electron characteristics of a metal in the high pressure region, Sov. Phys. JETP 11, 1130 (1960), http://www.jetp.ac.ru/cgi-bin/dn/e_011_05_1130.pdf.

[26] K. Kim, M. Yankowitz, B. Fallahazad, S. Kang, H. C. P. Movva, S. Huang, S. Larentis, C. M. Corbet, T. Taniguchi,
K. Watanabe, S. K. Banerjee, B. J. LeRoy, and E. Tutuc, van der Waals heterostructures with high accuracy rotational alignment, Nano Lett. 16, 1989 (2016).

[27] P. Rickhaus, F. K. de Vries, J. Zhu, E. Portolés, G. Zheng, M. Masseroni, A. Kurzmann, T. Taniguchi, K. Wantanabe, A. H. MacDonald, T. Ihn, and K. Ensslin, Density-wave states in twisted double-bilayer graphene, arXiv:2005.05373.

[28] C. De Beule, P. Silvestrov, M.-H. Liu, and P. Recher, Valley splitter and transverse valley focusing in twisted bilayer graphene, arXiv:1912.03191.

[29] Y. S. Ang, S. A. Yang, C. Zhang, Z. Ma, and L. K. Ang, Valleytronics in merging Dirac cones: All-electriccontrolled valley filter, valve, and universal reversible logic gate, Phys. Rev. B 96, 245410 (2017).

[30] ETH Zurich research collection, https://doi.org/10.3929/ ethz-b-000438922. 